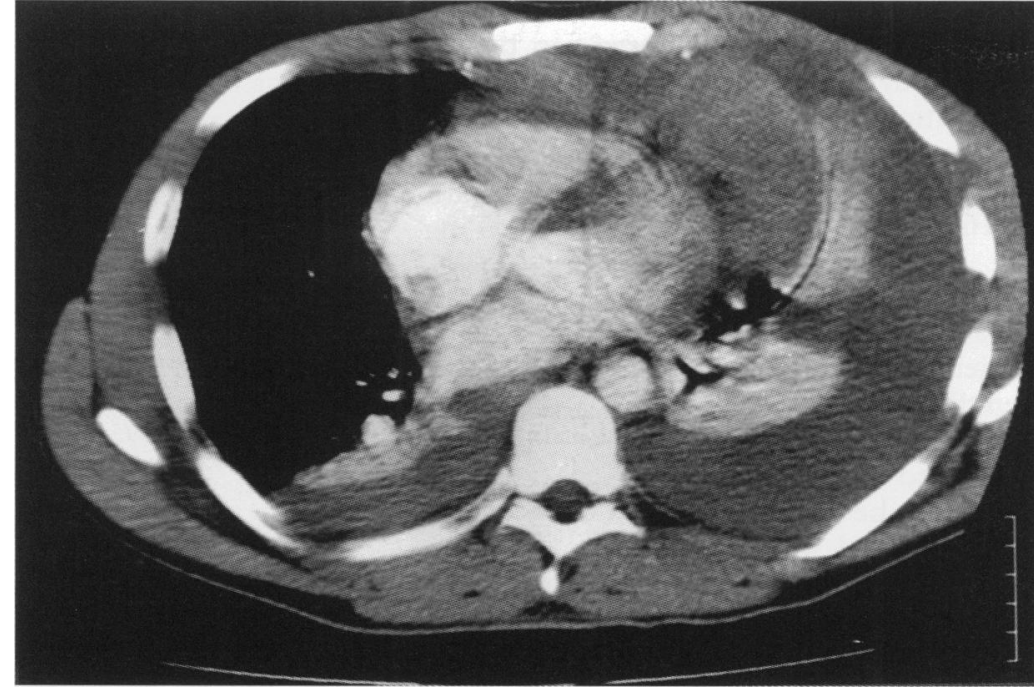

Figure 1 Computed tomogram of the chest.

tree, which showed no evidence of tears or fresh blood. A left chest drain was inserted with immediate drainage of $1600 \mathrm{ml}$ of blood. The drain was removed after three days, with a chest radiograph before discharge (eight days) showing regression of the mediastinal haematoma and expansion of the lung.

In this case, despite the moderate trauma, both the chest radiography and computed tomography revealed considerable mediastinal haemorrhage, and we feel that the underlying pathophysiology may have been a direct blow causing deformation of the sternum without fracture, leading to tearing of mediastinal vessels, possibly the internal mammary vein or artery. Other reported complications of blunt chest trauma include: direct cardiac injury with cardiac rupture, left ventricular aneurysms, or pseudoaneurysms $^{1}$; valve incompetence ${ }^{2}$; and damage to the thoracic duct. ${ }^{3}$ Damage to the internal mammary artery has been reported, with the formation of an anterior mediastinal haematoma producing external pericardial compression requiring surgical intervention. ${ }^{4}$ There is also a small incidence of blunt chest trauma induced cardiac arrest during sports activities (commotio cordis) in young athletes with a relatively poor survival rate due to delayed resuscitation. ${ }^{5}$ We have presented a case of considerable mediastinal haemorrhage after a rugby tackle associated with no bony injuries. This case demonstrates that even if the trauma appears trivial, adequate investigation is the only method for excluding potentially life threatening complications.

1 Maselli D, Micalizzi E, Pizio R, et al. Posttraumatic left ventricular pseudoaneurysm due to intramyocardial dissecting haematoma. Ann Thorac Surg 1997;64:830-1.

2 Banning A, Durrani A, Pillai R. Rupture of the atrial septum and tricuspid valve after blunt chest trauma. Ann Thorac Surg 1997;64:240-2.

3 Ikonomidis J, Boulanger B, Brenneman F. Chylothorax after blunt chest trauma: a report of 2 cases. Can $\mathcal{f}$ Surg 1997;40:135-8.

4 Irgau I, Fulda G, Hailstone D, et al. Internal mammary artery injury, anterior mediastinal hematoma, and cardiac 1018-21.

5 Maron B, Strasburger J, Kugler J, et al. Survival following blunt chest impact-induced cardiac arrest during sports activities in young athletes. Am f Cardiol 1997;79:840-1.
Department of Surgery, University Hospital Ghent, Belgium

J J De Waele

F E G Vermassen

Correspondence to: Dr Jan J De Waele,

Weezenhof 3242, $6536 \mathrm{GL}$ Nijmegen, The Netherlands (e-mail: jan.dewaele@) rug.ac.be).

\title{
Splenic herniation causing massive haemothorax after blunt trauma
}

\author{
Jan J De Waele, Frank E G Vermassen
}

Massive haemothorax always focuses attention to the chest and intrathoracic causes of haemodynamic instability. If such injuries are not recognised in the emergency room, potential for survival is limited.

A 45 year old male was admitted to the emergency department. He was an unrestrained passenger in a motor vehicle crash. On admission, he was haemodynamically unstable, and resuscitation according to Advanced Trauma Life Support guidelines started. He was tachypnoeic (25 breaths/min) and subcutaneous emphysema was present over the chest. Chest radiography revealed four rib fractures and haemothorax on the left side, with five rib fractures on the right. Chest tubes were inserted on both the left and right, and orotracheal intubation was necessary because of respiratory insufficiency. Ultrasound on admission could not detect any free intraperitoneal fluid. The left thoracostomy tube drained an initial volume of $1200 \mathrm{ml}$ fresh blood, and continued to produce about 200 $\mathrm{ml} / 5$ min (fig 1). Despite aggresive fluid therapy, the patient's haemodynamics deteriorated, and the patient was transferred to theatre. At operation, $3000 \mathrm{ml}$ blood was aspirated from the left hemithorax. There was a $4 \mathrm{~cm}$ tear in the diaphragm. The uninjured

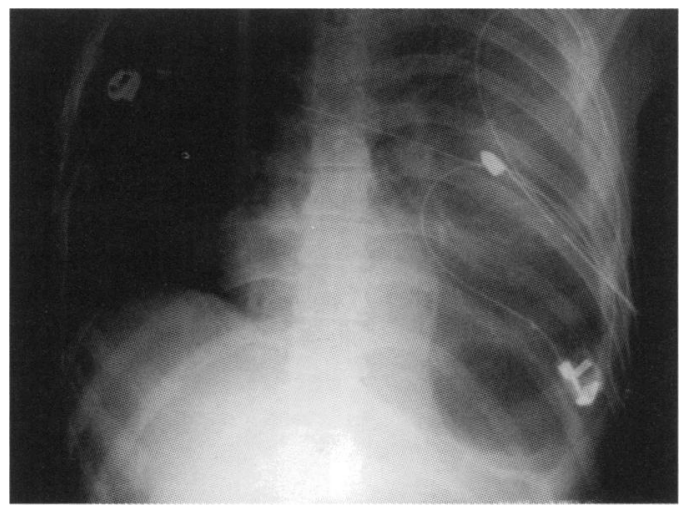

Figure 1 Chest radiograph after bilateral chest tube placement, and orotracheal intubation. 
splenic hilum sealed it, and functioned as a one way valve. The spleen was herniated in the thorax, completely disrupted, and bled profoundly. The diaphragmatic tear was extended and the spleen resected through the diaphragm. The patient recovered well, and was discharged on the 16 th postoperative day.

When haemodynamic instability persists after aggressive fluid replacement, surgery should not be deferred. In patients with thoracoabdominal trauma, it is often difficult to decide which side of the diaphragm should be considered first. In these cases focused abdominal ultrasound in trauma (FAST) can be a useful examination, as it can be performed at the bedside during initial resuscitation. Large amounts of blood both in the abdomen and thorax can be diagnosed with high accuracy. ${ }^{1}$ Diaphragmatic injury remains a difficult diagnosis in the multiply injured patient, ${ }^{23}$ and this case shows that it can profoundly change the clinical presentation of a common injury, such as splenic trauma. Whenever haemodynamic instability is present, consideration of potential diaphragmatic injuries should not defer prompt and adequate treatment.

1 Rothlin MA, Naf R, Amgwerd M, et al. Ultrasound in blunt abdominal and thoracic trauma. $\mathcal{F}$ Trauma 1993;34:48895.

2 Morgan AS, Flancbaum L, Esposito T, et al. Blunt injury to the diaphragm: an analysis of 44 patients. $\mathcal{f}$ Trauma 1986;26:565-8.

3 Shah R, Sabanathan S, Mearns AJ, et al. Traumatic rupture of the diaphragm. Ann Thorac Surg 1995;60:1444-9.

\title{
Basal skull fracture with intracranial air
}

\author{
R S Moore
}

A 49 year old man was brought to the accident and emergency department with a Glasgow coma score of 10 and bleeding from his right ear. A convergent squint was evident from inturning of the right eye. His head had been inadvertently crushed between a metal trolley and a wall. There were no other injuries. After resuscitation according to Advanced Trauma Life Support guidelines, radiography of his cervical spine demonstrated free air around the spinal cord (fig 1). The basal skull fracture with intracranial air was duly confirmed a short time later with bone windows on computed tomography (fig 2). The patient made a slow

\section{Department of Accident and Emergency Medicine, Northampton General Hospital NHS Trust, Cliftonville, Northampton NN1 5BD}

Correspondence to: Dr Moore, Consultant in Accident and Emergency Medicine.

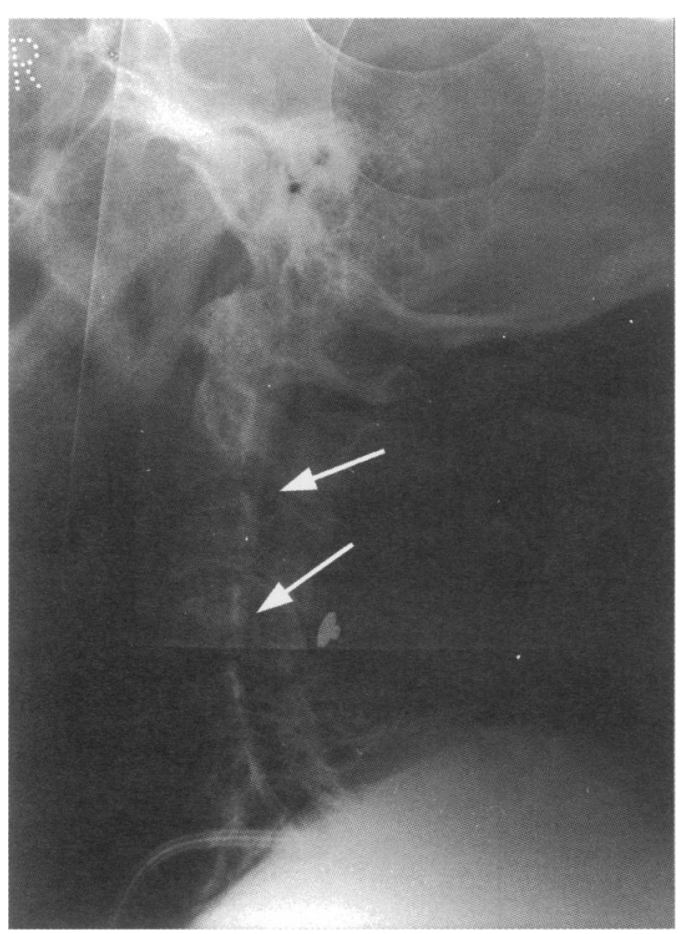

Figure 1 Radiograph of the cervical spine; the arrows indicate the air as a dark streak. but uneventful recovery after a period of observation with antibiotic prophylaxis. He retains a minor squint.

This case highlights the value of a standardised approach to the management of major trauma and the need to be vigilant for the unusual when looking at any $x$ ray film. Intracranial air after head injury has been the subject of medical reports since $1884^{1}$ and was initially thought to be rare, but Briggs drew attention to the problem, highlighting an incidence of $0.2 \%$ in all head injuries. ${ }^{2}$ Orebaugh and Margolis later placed the incidence as between $0.5 \%$ and $1 \%{ }^{3}$ The majority of these cases involved injuries to the face and gave rise to cerebrospinal fluid (CSF) rhinorrhoea. The

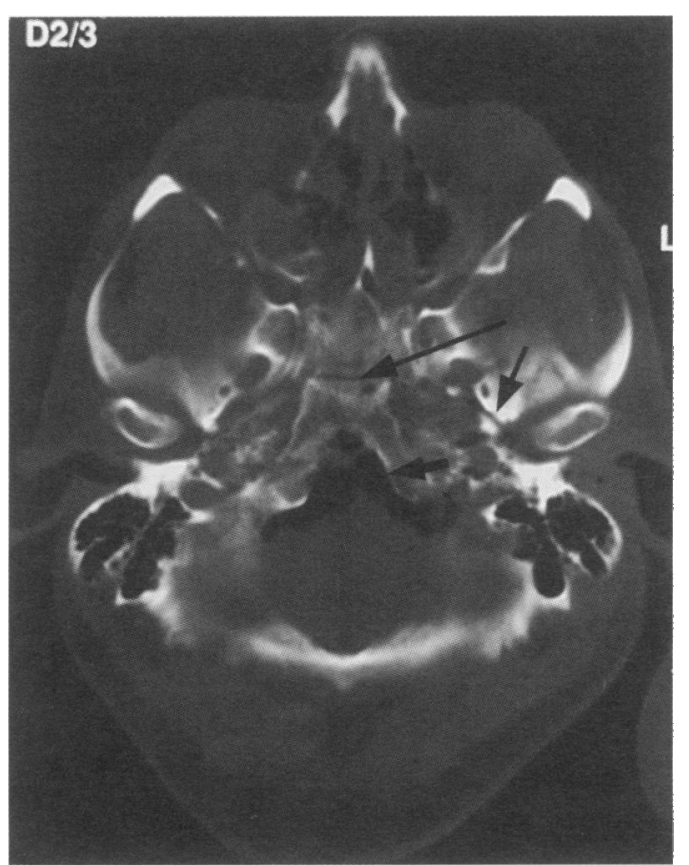

Figure 2 Computed tomography of the head showing the fracture (thin arrows) and free air (thick arrow). 